TITLE:

\title{
Multiple first-order metamagnetic transitions and quantum oscillations in ultrapure Sr3Ru2O7
}

\author{
AUTHOR(S): \\ Perry, RS; Kitagawa, K; Grigera, SA; Borzi, RA; \\ Mackenzie, AP; Ishida, K; Maeno, Y
}

\section{CITATION:}

Perry, RS ... [et al]. Multiple first-order metamagnetic transitions and quantum oscillations in ultrapure Sr3Ru207. PHYSICAL REVIEW LETTERS 2004, 92(16): 166602.

\section{ISSUE DATE:}

2004-04-23

URL:

http://hdl.handle.net/2433/49956

RIGHT:

Copyright 2004 American Physical Society 


\title{
Multiple First-Order Metamagnetic Transitions and Quantum Oscillations in Ultrapure $\mathrm{Sr}_{3} \mathrm{Ru}_{2} \mathrm{O}_{7}$
}

\author{
R. S. Perry, ${ }^{1,2}$ K. Kitagawa, ${ }^{2}$ S. A. Grigera,${ }^{3}$ R. A. Borzi, ${ }^{3}$ A. P. Mackenzie,${ }^{3}$ K. Ishida, ${ }^{2}$ and Y. Maeno ${ }^{1,2}$ \\ ${ }^{1}$ Kyoto University International Innovation Center, Kyoto 606-8501, Japan \\ ${ }^{2}$ Department of Physics, Kyoto University, Kyoto 606-8502, Japan \\ ${ }^{3}$ School of Physics and Astronomy, University of St. Andrews, St. Andrews KY16 9SS, United Kingdom
}

(Received 10 September 2003; published 23 April 2004)

\begin{abstract}
We present measurements on ultraclean single crystals of the bilayered ruthenate metal $\operatorname{Sr}_{3} \mathrm{Ru}_{2} \mathrm{O}_{7}$, which has a magnetic-field-tuned quantum critical point. Quantum oscillations of differing frequencies can be seen in the resistivity both below and above its metamagnetic transition. This frequency shift corresponds to a small change in the Fermi surface volume that is qualitatively consistent with the small moment change in the magnetization across the metamagnetic transition. Very near the metamagnetic field, unusual behavior is seen. There is a strong enhancement of the resistivity in a narrow field window, with a minimum in the resistivity as a function of temperature below $1 \mathrm{~K}$ that becomes more pronounced as the disorder level decreases. The region of anomalous behavior is bounded at low temperatures by two first-order phase transitions. The implications of the results are discussed.
\end{abstract}

DOI: 10.1103/PhysRevLett.92.166602

PACS numbers: 72.15.-v, 68.35.Rh, 71.27.+a, 74.70.Pq

Magnetic field tuning of quantum critical points in itinerant electron systems has generated considerable interest in recent years. The effect of field-tuned fluctuations has been studied in a number of systems including $\mathrm{URu}_{2} \mathrm{Si}_{2}$ [1,2], $\mathrm{YbRh}_{2} \mathrm{Si}_{2}$ [3], $\mathrm{CeRu}_{2} \mathrm{Si}_{2}$ [4,5], $\mathrm{UPt}_{3}$ [6], and $\mathrm{Fe} / \mathrm{Cr}$ multilayers [7]. In most studied systems, the quantum critical point results from the "standard method" of using the field to depress the transition temperature of a second order phase transition. A condition for this approach is that the symmetry of the low-field phase be different from that of a field-polarised paramagnet. Recently, it has also been noted that quantum criticality can result in situations such as itinerant electron metamagnets in which both high- and low-field phases have the same symmetry [8]. In this case, the quantum critical point is the end point of a line of symmetry-conserving first-order transitions $[8,9]$.

The material that first stimulated the discussion of the quantum critical end point is the subject of this Letter, the bilayer ruthenate $\mathrm{Sr}_{3} \mathrm{Ru}_{2} \mathrm{O}_{7}$. It is the $n=2$ member of the Ruddlesden-Popper series of layered perovskites $\mathrm{Sr}_{n+1} \mathrm{Ru}_{n} \mathrm{O}_{3 n+1}$ whose properties are dominated by layers of ruthenium oxide octahedra. The four electrons in the ruthenium $4 d t_{2 g}$ orbitals hybridise with the oxygen $2 p$ to create metallic bands that are narrow but still sufficiently broad to avoid insulating transitions in the majority of the materials. High purity was first reached in the $n=1$ member of the series, $\mathrm{Sr}_{2} \mathrm{RuO}_{4}$, enabling the observation of an unconventional superconducting state [10] in which there is good evidence for spin triplet pairing [11]. As the purity of $\mathrm{Sr}_{3} \mathrm{Ru}_{2} \mathrm{O}_{7}$ was improved, it was realized that its ground state was paramagnetic [12] rather than ferromagnetic as had initially been reported [13]. High-field studies then revealed the phenomenon of metamagnetism, i.e., a sudden superlinear rise in magnetization as a function of applied magnetic field [14]. Analysis of transport and specific heat gave evidence for quantum critical fluctuations. However, additional anomalous behavior in the temperature dependent resistivity was observed very near to the critical field $H_{c}$ for magnetic fields applied parallel to the crystallographic $c$ axis $[8,14]$. We concentrate on this orientation of the field throughout the present Letter.

The anomaly seen near $H_{c}$ was a small change to the temperature dependent part of the resistivity. Very near to quantum critical points, the resistivity in itinerant systems often varies as $T^{\alpha}$, where the exponent $\alpha$ crosses over from some value less than two to become equal to two as Fermi liquid properties are recovered below some characteristic temperature $T^{*}$ [15]. In $\mathrm{Sr}_{3} \mathrm{Ru}_{2} \mathrm{O}_{7}$, a value of $\alpha$ higher than two was seen below $0.8 \mathrm{~K}$ for fields $\mu_{0} H_{c} \pm 0.05 T$ [8] (see also inset of Fig. 1). This observation was surprising because it could not be a simple consequence of critical fluctuations, but the experimental signal was very small. Many questions therefore remain open. Do the observations represent a violation of Matthiessen's rule in the presence of a strongly peaking resistivity, or are they the first hint of more profoundly unusual behavior near $H_{c}$ ? A crucial issue, of relevance far beyond $\mathrm{Sr}_{3} \mathrm{Ru}_{2} \mathrm{O}_{7}$, is the role of disorder near metallic quantum critical points $[15,16]$. In a naïve approach, the most obvious way to study this would be via the deliberate introduction of disorder, since the existing crystals of $\mathrm{Sr}_{3} \mathrm{Ru}_{2} \mathrm{O}_{7}$ were clean by the standards of the field, with values of the residual resistivity $\left(\rho_{\text {res }}\right)$ of less than $3 \mu \Omega \mathrm{cm}$. Ruthenate physics is, however, known to be extremely sensitive to disorder [17], so we have taken the opposite approach of striving to decrease the levels 


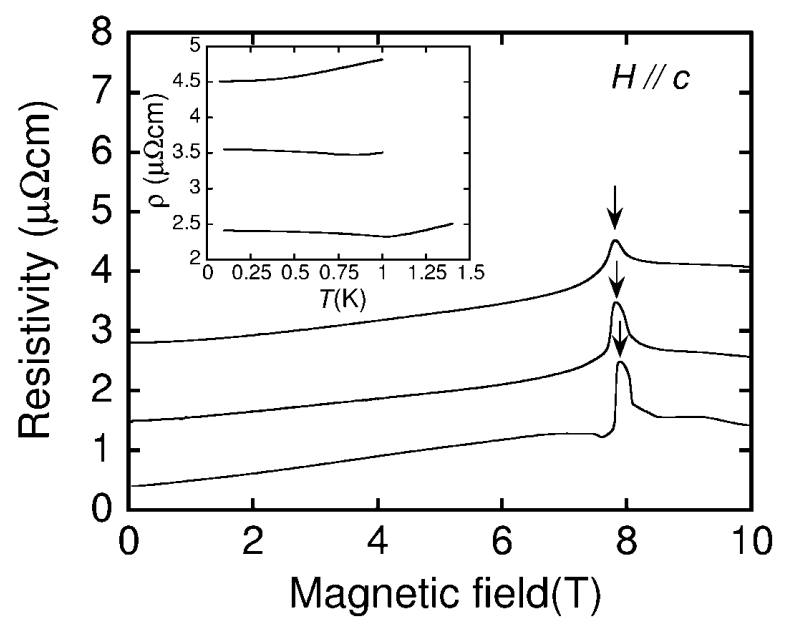

FIG. 1. The resistivity ( $\rho)$ versus magnetic field in single crystals of $\mathrm{Sr}_{3} \mathrm{Ru}_{2} \mathrm{O}_{7}$ for different values of residual resistivity $\rho_{\text {res }}$. The temperature of each curve is between 0.06 and $0.1 \mathrm{~K}$, and the magnetic field is perpendicular to the $\mathrm{RuO}_{2}$ planes. Inset: Temperature sweeps at the fields indicated by the arrows in each case.

of disorder. As we will show, this has resulted in remarkable qualitative changes in the behavior near the metamagnetic field.

The $\mathrm{Sr}_{3} \mathrm{Ru}_{2} \mathrm{O}_{7}$ crystals used for this work were grown using a floating zone technique at Kyoto and measurements were made on several crystals from different growth batches at both Kyoto and St. Andrews Universities. In Fig. 1 we show the effect on the magnetoresistivity of decreasing $\rho_{\text {res }}$ from 2.8 to $0.4 \mu \Omega \mathrm{cm}$. For this direction of the applied field (parallel to the crystal$\log$ raphic $c$ axis), a single peak is seen in the magnetoresistance for $\rho_{\text {res }}=2.8 \mu \Omega \mathrm{cm}$. As the sample purity increases, the same basic peak remains as the dominant feature, but more structure develops. In particular, there is a pronounced dip at 7.5 T, and the central peak is seen to have very steep sides at 7.8 and $8.1 T$. The profound effect that decreasing the disorder levels is having is emphasized by the data shown in the inset of Fig. 1, which shows the result of sweeping the temperature at fixed fields chosen to be at the resistive maximum for each purity level. In the inset, the top trace shows the unusual $T^{\sim 3}$ dependence previously reported for $\rho_{\text {res }}=2.8 \mu \Omega \mathrm{cm}$ [8]. As the samples become purer, the temperature dependence of $\rho$ at the metamagnetic transition actually changes to a negative gradient at low temperatures, with a minimum that increases systematically in temperature. The anomalous flattening of $\rho$ previously observed can now be accounted for as the onset of a very weak minimum that has been depressed by the disorder. A set of temperature sweeps at fixed field for one of our purest samples is presented in Fig. 2 to emphasize that the region of the $(H, T)$ plane in which the strange behavior is seen is extremely small. Above $1.2 \mathrm{~K}, \rho$ depends only weakly on field. Below that temperature, however, the behavior at the peak differs remarkably from that on either side.

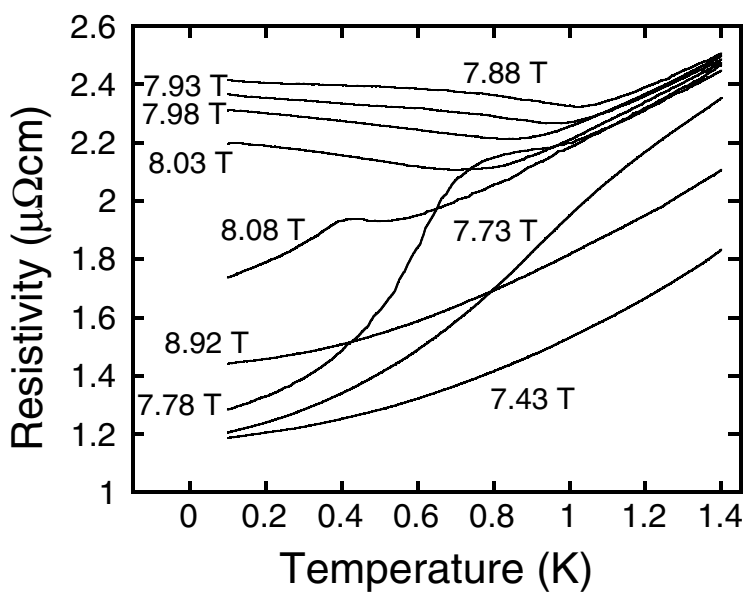

FIG. 2. Temperature sweeps of the resistivity at magnetic fields near the metamagnetic transition. The crystal has $\rho_{\text {res }}=0.55 \mu \Omega \mathrm{cm}$, and the sweep rate was approximately $30 \mathrm{mK} / \mathrm{min}$. The measurements were made at Kyoto University, with the samples mounted inside the mixing chamber of a dilution refrigerator to ensure good thermal contact between the samples and the thermometer.

Below 7.8 and above $8.1 T, \rho$ has a positive gradient all the way down to $50 \mathrm{mK}$, crossing over to $T^{2}$ behavior similar to that previously reported [8]. In between these fields, however, a clear negative gradient is seen at low temperatures.

The almost steplike changes in $\rho(H)$ shown for the purest sample in Fig. 1 are reminiscent of the features expected at a first-order phase transition. To investigate them more in depth, we performed a study of the ac magnetic susceptibility $\chi(\omega)$. As discussed in Ref. [18], $\chi(\omega)$ is a sensitive probe for hysteretic dissipation through the appearance of an imaginary part $\chi^{\prime \prime}(\omega)$. The study of the lower quality crystals had shown that for an applied field in the crystallographic $a b$ plane, a single first-order transition line terminated in a critical point at approximately $1.25 \mathrm{~K}$. As the field was rotated towards the $c$ axis, the end point temperature was smoothly depressed to below $50 \mathrm{mK}$ at around $10^{\circ}$ from the $c$ axis. No peak in $\chi^{\prime \prime}$ was observable for the field parallel to the $c$ axis. Like $\rho, \chi$ in the purest samples differs markedly from that in the previous generation of crystals near the metamagnetic field [17]. $\rho$ and $\chi$ of samples with $\rho_{\text {res }}<$ $0.5 \mu \Omega \mathrm{cm}$ are shown in Fig. 3. It should be noted that the two steep features in $\rho$ coincide with peaks in both $\chi^{\prime}$ and $\chi^{\prime \prime}$. The peaks in $\chi^{\prime \prime}$ are particularly significant, as they indicate the presence of dissipation that is most easily interpreted in terms of hysteresis at a first-order phase boundary. The data shown were taken at a temperature of $20 \mathrm{mK}$, but a series of sweeps at constant temperature in steps of $100 \mathrm{mK}$ established that the dissipative peaks at 7.8 and $8.1 \mathrm{~T}$ are observable up to $\sim 0.8$ and $\sim 0.4 \mathrm{~K}$, respectively. Another apparent correlation between $\rho$ and $\chi$ is between a peak in $\chi^{\prime}$ and onset of a dip in resistivity at $\sim 7.5 \mathrm{~T}$. 


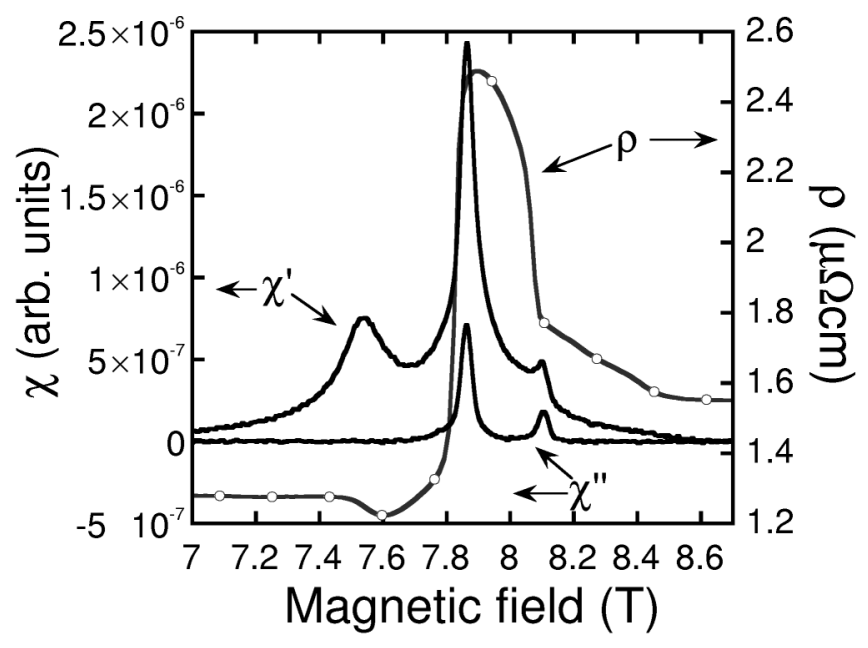

FIG. 3. The susceptibility $(\chi)$ and resistivity $(\rho)$ versus magnetic field across the metamagnetic field. The measurements were made at $T=20 \mathrm{mK}$ on different crystals, both having $\rho_{\text {res }}<0.5 \mu \Omega \mathrm{cm}$. The ac excitation field was $0.32 \mathrm{G}$ at a frequency of $83.4 \mathrm{~Hz}$.

A summary of the findings presented in Figs. 1 and 3 is therefore as follows. Remarkably, decreasing the levels of disorder leads to a negative gradient in $\rho(T)$ near the metamagnetic field and to the resolution of several pronounced features in $\rho$ instead of the single peak seen in samples with $\rho_{\text {res }}=2.8 \mu \Omega \mathrm{cm}$. In $\chi$, three peaks are seen at low $T$, two of which signal the crossing of firstorder phase boundaries.

At first sight, these observations question the use of pictures based on itinerant electron magnetism [19] as the correct starting point for considering the physics of $\mathrm{Sr}_{3} \mathrm{Ru}_{2} \mathrm{O}_{7}$. The basic idea of such theories is that the moment change at the metamagnetic field is due to a field-induced nonlinear exchange split of the Fermi surface. The consequence of this should be a change of Fermi surface topography between the low-field and high-field states due to differences in the spin-up and spin-down
Fermi volumes, but without a large overall change in total Fermi volume. The dramatically improved purity of the new samples allows us to address this issue via the first observation of quantum oscillations reported for $\mathrm{Sr}_{3} \mathrm{Ru}_{2} \mathrm{O}_{7}$. In Fig. 4 we show tiny Shubnikhov-de Haas oscillations (less than $0.2 \%$ of the total resistivity) from the field regions above and below the metamagnetic transition. Frequencies corresponding to large Fermi surface sheets are observed in both cases, and all observed frequencies undergo changes of $\pm 10-30 \%$ across the metamagnetic transition [20]. The Fermi surface of $\mathrm{Sr}_{3} \mathrm{Ru}_{2} \mathrm{O}_{7}$ is likely to be complicated [21], so a full analysis of the quantum oscillations is a separate project. We note that quantum oscillations were observed for the magnetic field off axis in the susceptibility and more indepth study is currently in progress. The results presented here already give strong support for the basic starting point of itinerant magnetism $[8,9,15,18,19]$ because the size of the relative volume changes seems entirely consistent with the low moment change at the metamagnetic transition of approximately $0.25 \mu_{B} / \mathrm{Ru}$ [22].

Although the underlying picture of physics based on itinerant electron metamagnetism is appropriate for $\mathrm{Sr}_{3} \mathrm{Ru}_{2} \mathrm{O}_{7}$, the detailed behavior near $H_{c}$ is not consistent with the simplest theories that are based only on a quantum critical end point. The key experimental findings are that the enhancement of $\rho$ in the metamagnetic region grows as the disorder scattering decreases and that the narrow field range over which the residual resistivity is enhanced seems to be bounded at either side by first-order phase transitions. Neither of these is predicted by, for example, Ref. [15]. Enhanced scattering at first-order phase transitions in metallic systems has been seen in other situations, notably in low carrier density materials such as quantum Hall ferromagnets [23]. In these systems, there are multiple peaks in the resistivity as a function of magnetic field corresponding to the crossing of Landau levels; in $\mathrm{Sr}_{3} \mathrm{Ru}_{2} \mathrm{O}_{7}$ we observe only one peak. Also in contrast with those observations, the resistivity in
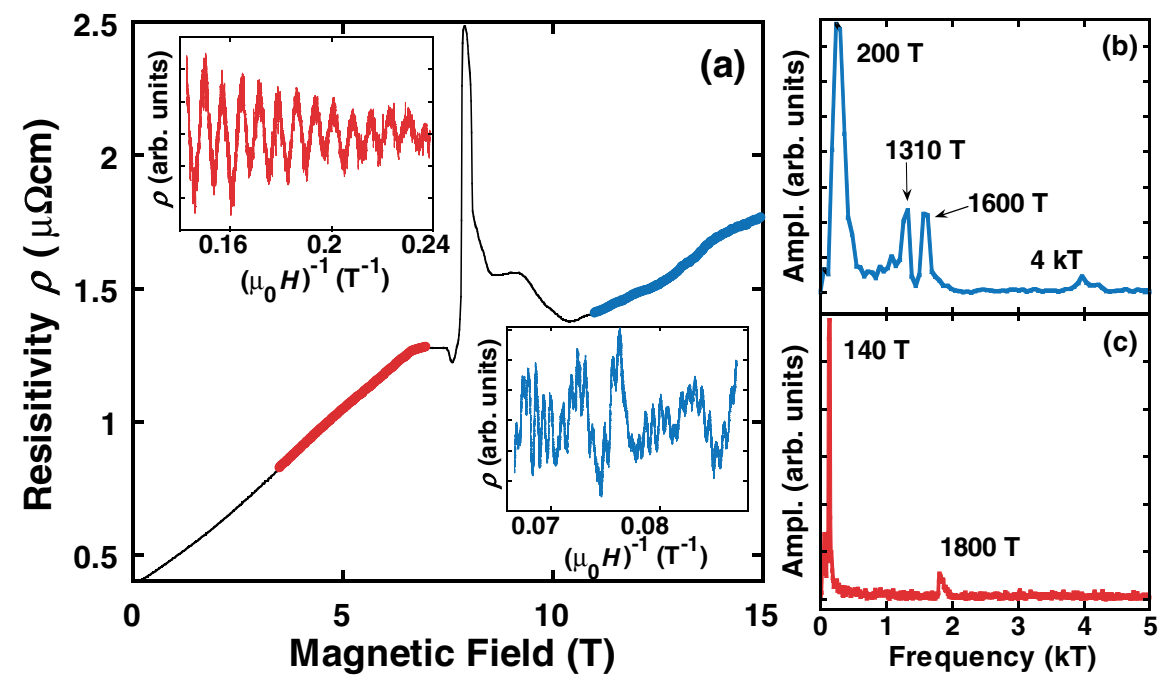

FIG. 4 (color). Shubnikov-de Haas oscillations in the resistivity of $\mathrm{Sr}_{3} \mathrm{Ru}_{2} \mathrm{O}_{7}$ in a crystal with $\rho_{\text {res }}=0.4 \mu \Omega \mathrm{cm}$. (a) The raw data with background subtraction in the insets for the low field (red) and high field (blue) regions, respectively. (b) and (c) show color coded Fourier transforms of the inset data giving valuable information on the Fermi surface of the metal. Similar frequencies were observed in both the 0.4 and $0.55 \mu \Omega \mathrm{cm}$ crystals. 
this case remains high in the field range between the two transitions. Qualitatively, the magnetoresistance has similarity to that of graphite as it undergoes a fielddependent charge density wave transformation over a finite range of applied fields [24]. There, however, the change of resistivity is due to a large reduction in the Fermi volume in the density wave state, and there seems to be no reason to expect such a state in $\mathrm{Sr}_{3} \mathrm{Ru}_{2} \mathrm{O}_{7}$. We do not resolve quantum oscillations in the region 7.8 to $8.1 \mathrm{~T}$, but in the high-field state the Fermi volume seems to have made, at best, a small change from that at low fields. We also note that the resistivity plateau could be due to a change in the Fermi velocity close to the critical field.

We currently propose no definite interpretation for the behavior near $H_{c}$, but are interested in further investigation of several possibilities. The first is that the data signal the entry of the system into some new ordered state that it adopts to avoid the consequences of the divergent fluctuations near the critical point. In this sense, the physics of the critical point dictates the behavior of the system, but theories based purely on the consequences of the associated fluctuations would be valid only outside the field range very near the metamagnetic transition. This reasoning (and the evidence for it) has been discussed in relation to $\mathrm{Sr}_{3} \mathrm{Ru}_{2} \mathrm{O}_{7}$ [8] and $\mathrm{URu}_{2} \mathrm{Si}_{2}$ [1,2]. Another possibility is that the first-order physics now being seen in the best samples indicates the presence of a Griffithslike phase [25] in which the high resistivity is dominated throughout the peak by the presence of magnetic domains. We see two distinct peaks in $\chi^{\prime \prime}$, but it may be possible to account for that in such a picture. Also, we cannot rule out the presence of a small dissipative signal or even static domains in the intermediate region. We plan to test for this in future with experiments involving field modulation of the resistivity.

In summary, we have reported a series of measurements on extremely pure single crystals of $\mathrm{Sr}_{3} \mathrm{Ru}_{2} \mathrm{O}_{7}$ with $\rho_{\text {res }}$ as low as $0.4 \mu \Omega \mathrm{cm}$. The extreme purity of the samples has enabled the observation of quantum oscillations both above and below the metamagnetic field $H_{c}$. More surprisingly, it has also highlighted intriguing qualitative changes to the behavior very near $H_{c}$. In the best samples, a well-defined region bounded by first-order phase transitions can be seen at low temperatures. Within this region, an upturn in $\rho(T)$ is observed below $1 \mathrm{~K}$. We believe that this provides a striking example of the extreme sensitivity of correlated electron phenomena to disorder in itinerant systems. It is sobering to realize that the previous generation of $\mathrm{Sr}_{3} \mathrm{Ru}_{2} \mathrm{O}_{7}$ crystals, in which the physics reported here was almost entirely unobservable, were already pure in comparison to many of the materials currently being used to study quantum criticality and its consequences.

We are pleased to acknowledge useful discussions with H. Fukazawa, A. G. Green, S. R. Julian, N. Kikugawa, G. G. Lonzarich, T. Sakakibara, L. Taillefer, T. Tayama,
H. Yaguchi, S. Nakatsuji, Kosaku Yamada, and S. Fujimoto. We also thank the Japan Society for the Promotion of Science, the Leverhulme Trust, the UK Engineering and Physical Science Research Council, and the Royal Society for valuable financial support. This work has also been supported by Grants-in-Aid for Scientific Research from the Japan Society for Promotion of Science and from the Ministry of Education, Culture, Sports, Science, and Technology (MEXT), and by the 21COE Program "Center for Diversity and Universality in Physics" from MEXT of Japan.

[1] M. Jaime et al., Phys. Rev. Lett. 89, 287201 (2002).

[2] N. Harrison, M. Jaime, and J. A. Mydosh, Phys. Rev. Lett. 90, 96402 (2003).

[3] P. Gegenwart et al., Phys. Rev. Lett. 89, 056402 (2002).

[4] H. Aoki et al., J. Phys. Soc. Jpn. 70, 774 (2001), and references therein.

[5] J. Flouquet et al., Physica (Amsterdam) 319B, 251 (2002), and references therein.

[6] J. S. Kim et al., Solid State Commun. 114, 413 (2000); G. R. Stewart, Rev. Mod. Phys. 73, 797 (2001), and references therein.

[7] F. G. Aliev, V.V. Moshchalkov, and Y. Bruynseraede, Phys. Rev. Lett. 81, 5884 (1998).

[8] S. A. Grigera et al., Science 294, 329 (2001).

[9] A. J. Millis et al., Phys. Rev. Lett. 88, 217204 (2002).

[10] Y. Maeno et al., Nature (London) 372, 532 (1994).

[11] A. P. Mackenzie and Y. Maeno, Rev. Mod. Phys. 75, 657 (2003).

[12] S. Ikeda et al., Phys. Rev. B 62, R6089 (2000).

[13] G. Cao et al., Phys. Rev. B 56, 5387 (1997).

[14] R. S. Perry et al., Phys. Rev. Lett. 86, 2661 (2001).

[15] Yong Baek Kim and A. J. Millis, Phys. Rev. B 67, 085102 (2003).

[16] A. Rosch, Phys. Rev. Lett. 82, 4280 (1999).

[17] L. Capogna et al., Phys. Rev. Lett. 88, 076602 (2002).

[18] S. A. Grigera et al., Phys. Rev. B 67, 214427 (2003).

[19] E. P. Wohlfarth and P. Rhodes, Philos. Mag. 7, 1817 (1962).

[20] The $4 \mathrm{k} T$ frequency only observed at high fields in our resistivity data has also been observed below the metamagnetic field in de Haas van Alphen experiments, with a slightly higher frequency [R. A. Borzi et al. (unpublished)].

[21] D. J. Singh and I. I. Mazin, Phys. Rev. B 63, 165101 (2001).

[22] Although the existence of multiple peaks in $\chi$ is often thought to result from local physics, we remark that it is also entirely consistent with the itinerant electron picture, in which it could result from multiple peaks in the density of states very near the Fermi level.

[23] E. P. De Poortere et al., Science 290, 1546 (2000).

[24] H. Yaguchi and J. Singleton, Phys. Rev. Lett. 81, 5193 (1998).

[25] R. B. Griffiths, Phys. Rev. Lett. 23, 17 (1969). 